\title{
TECNOLOGIA MÓVEL UTILIZADA NO INVENTÁRIO FLORESTAL
}

\author{
Catize Brandelero ${ }^{1}$, Enio Giotto ${ }^{2}$, Luciano Farinha Watzlavick ${ }^{3}$, \\ Rudiney Soares Pereira ${ }^{2}$, Salete Andreis ${ }^{4}$ \\ ${ }^{1}$ Eng $^{\mathrm{a}}$. Florestal, Doutoranda em Engenharia Florestal, UFSM, Santa Maria, RS, Brasil - catizebrandelero@gmail.com \\ ${ }^{2}$ Eng. Florestal, Dr., Depto. de Engenharia Rural, UFSM, Santa Maria, RS, Brasil - giotto@ccr.ufsm.br - rudiney@smail.ufsm.br \\ ${ }^{3}$ Eng. Florestal, Dr., Depto. de Agronomia, UNICENTRO, Guarapuava, PR, Brasil - luciano.watzlawick@pq.cnpq.br \\ ${ }^{4}$ Eng $^{\mathrm{a}}$. Civil, Mestranda em Geomática, UFSM, Santa Maria, RS, Brasil - salete_andreis@hotmail.com \\ Recebido para publicação: 28/01/2008 - Aceito para publicação: 05/05/2008
}

\begin{abstract}
Resumo
Com o objetivo de agilizar os processamentos, diminuir custos e otimizar as atividades no processamento das informações oriundas dos inventários florestais, foi elaborado um software para aparelhos do tipo Pocket PC, desenvolvido com linguagem NSBasic e banco de dados moldado no MSAcess. A possibilidade de integração dos aparelhos do tipo Pocket PC com o GPS bluetooth torna possível a localização e o registro das coordenadas geográficas de localização das parcelas florestais. O software permite registrar os projetos, cadastrar as espécies florestais, cadastrar parcelas e registrar os elementos dendrométricos e fitossanitários, além de permitir calcular o volume $\left(\mathrm{m}^{3} / \mathrm{ha}\right)$ e a área basal média/ha. Dessa forma, os dados coletados em campo podem ser descarregados diretamente em planilhas para a realização do processamento do inventário florestal, em que serão geradas as informações quantitativas e qualitativas da floresta.
\end{abstract}

Palavras-chave: Quantificação florestal; Pocket PC; software.

\begin{abstract}
Mobile technology used in the forest inventory. Aiming to enhance the processes, decrease costs and optimize the activities in the forest inventory data processing, a software was developed for Pocket PC type of equipment, written in NSBasic language and data bank adjusted in the MSAcess. The possible integration between Pocket PC and Bluetooth GPS enables the geographical coordinates locating and register of the forest plots location. The software enables projects registration; forest species record; plots record; register plant sanitary and dendrometric elements in addition of enabling volume calculation $\left(\mathrm{m}^{3} / \mathrm{ha}\right)$ and average basal area/ha. This way the field collected data can be directly transfered to calculation sheets to make the forest inventory processing, where forest qualitative and quantitative data will be generated.

Keywords: Forest measurements; Pocket PC; software.
\end{abstract}

\section{INTRODUÇÃO}

Em virtude dos avanços humanos, a utilização dos recursos florestais foi essencial para o desenvolvimento regional e vem se acentuando com o grau de crescimento das populações. Esse aumento da demanda por esses produtos exige cada vez mais o emprego de práticas de manejo para se obter aumento da produção. Para que isso ocorra, o inventário florestal é uma ferramenta primordial para o desenvolvimento de tecnologias no setor, pois alimenta os sistemas de informações geográficas com as informações oriundas do campo.

O levantamento e a qualificação do potencial de produção florestal não são efetivos, pois demandam elevado fluxo de informações, criando dificuldades para alocar, detalhar, organizar, interpretar e principalmente armazenar os dados de forma que garantam um confiável banco de dados para tomada de decisões técnico-administrativas.

No entanto, torna-se possível sistematizar tais informações através do desenvolvimento das tecnologias computacionais (hardwares e softwares) aliadas à tecnologia GPS (Global Positioning System) e à fabricação de circuitos integrados que possuem um novo modelo - o paradigma da mobilidade -, facilitando o desenvolvimento das atividades (MATEUS; LOUREIRO, 2004). 
A mobilidade possibilitada pela utilização da tecnologia bluetooth agiliza as conexões sem fio, à curta distância, e a comunicação entre vários dispositivos eletrônicos. A utilização desses dispositivos eletrônicos disponíveis, aliada à criação de softwares, tende a fornecer informações capazes de alimentar complexos bancos de dados que poderão servir de base para o planejamento adequado nos empreendimentos florestais.

Devido à necessidade de se efetuarem atividades silviculturais de forma mais ágil e eficiente, o objetivo deste trabalho é apresentar o sistema de coleta de dados para inventários florestais para florestas plantadas a partir do uso de Pocket PC e aparelho GPS com conexão bluetooth.

\section{REVISÃO BIBLIOGRÁFICA}

\section{Inventário florestal}

O processo de tomada de decisões sobre as diversas questões que envolvem a ciência florestal necessita de informação. Uma das formas de se obter informações quantitativas e qualitativas das florestas é dada pelo Inventário Florestal (IF), o qual, segundo Husch et al. (1972), consiste na aplicação de princípios de medição para se obterem informações quantitativas que produzem decisões. Já Péllico Netto; Brena (1997) conceituam o IF como a atividade que visa obter informações qualitativas e quantitativas dos recursos florestais existentes em uma área pré-especificada.

Seguindo a linha de desenvolvimento tecnológico aliado aos inventários florestais, pode-se mencionar que, durante o IF Nacional, realizado pelo antigo IBDF (Instituto Brasileiro de Desenvolvimento Florestal 1981/82 - estados do Paraná e Santa Catarina), foi coletado um número expressivo de dados. Isso ocasionou a necessidade de 40 programas computacionais para 0 processamento dos dados referentes às árvores cubadas e amostradas. Os dados foram perfurados em cartões a partir de formulários específicos e armazenados em discos magnéticos, obedecendo às estruturas requeridas pelo sistema (PÉLLICO NETTO, 1984).

Os inventários florestais eram executados dentro de padrões metodológicos e tecnológicos simples. Com o passar do tempo e a evolução tecnológica, passou-se a utilizar ferramentas sofisticadas de apoio. Dentre estas, pode ser citada a utilização de fotografias aéreas, de imagens (satélite, sensores aerotransportados e vídeo), a utilização do sistema GPS (Sistema de Posicionamento Global) e do SIG (Sistema de Informações Geográficas), assim como o desenvolvimento da computação, envolvendo toda a tecnologia da informação para processamento e análise dos dados levantados (SANQUETTA et al., 2006). Fazendo uso de um SIG, as informações podem ser armazenadas, atualizadas, recuperadas e associadas a mapas florestais, tornando mais fácil e rápido analisar e exibir graficamente os resultados (AVERY; BURKHART, 1994).

Atualmente, temos como referência o inventário florestal contínuo realizado em 2001, no estado do Rio Grande do Sul. Efetuado com o auxílio de modernas tecnologias e equipamentos, foram adotados sistemas computacionais específicos para a realização do mapeamento e formação do banco de dados. Essa base de informações está sendo utilizada para formulação de estratégias que garantam a sustentabilidade de matéria-prima de qualidade e ampliação do parque industrial (SEBEM, 2000).

Watzlawick et al. (2000) apresentam um exemplo concreto de aplicação do Sistema de Posicionamento Global (GPS) para fornecer a posição geográfica (latitude e longitude) em relação ao nível médio dos mares de parcelas amostrais em um inventário florestal, no qual o processo de amostragem utilizado foi o sistemático em dois estágios. Concluíram que a utilização do GPS, como ferramenta de localização, navegação e apoio ao inventário florestal, proporcionou melhor rendimento, bem como uma economia de tempo e de recursos na execução da atividade.

A grande utilização dessas tecnologias vem sendo feita nas mais diversas áreas dos levantamentos florestais, melhorando a logística e os custos. Para Sanquetta et al. (2006), o uso de tecnologias adequadas pode mais bem caracterizar sítios e superfícies. Pode-se realizar ainda a localização de uma vasta gama de informações para a caracterização momentânea ou para o monitoramento ambiental da área.

\section{Tecnologia móvel}

Tecnologias móveis são dispositivos computacionais que estão mudando a forma de realizar atividades quando estamos em movimento ou não desejamos ficar limitados a uma infra-estrutura (MATEUS; LOUREIRO, 1998). 
A mobilidade permite, assim, um acesso fácil e rápido à informação, devido à maior organização (HP, 2005). Ela tem como objetivo prover acesso permanente ao usuário em redes fixas ou móveis, independentemente de sua posição. As características da computação móvel são o tipo e a capacidade de processamento, a mobilidade do usuário e da unidade móvel e a comunicação com outro elemento computacional, por via bluetooth (LOUREIRO et al., 2003).

Os avanços ocorridos na área de microprocessadores, materiais de sensoriamento remoto, microsistemas eletromecânicos (MEMS - Micro Electro-Mecanical Systems) e comunicação sem fio, têm estimulado o desenvolvimento e o uso de sensores "inteligentes" em áreas ligadas a processos físicos, químicos e biológicos, entre outros (LOUREIRO et al., 2003).

A crescente migração da computação desktop-based para a computação móvel, disponibilizada por equipamentos como palmtops, handhelds e celulares com capacidade de integração com a Internet, representa uma importante mudança de paradigma (GARCIA; LIMA, 2003).

Essa comunicação em curta distância de redes sem fio, também chamada de conexão Bluetooth, é um sistema com baixo custo e alta operabilidade (KOBAYASHI, 2004; BILLO, 2003). A tecnologia visa à conectividade num sentido geral, envolvendo os equipamentos de uma área restrita, particularmente entre os "móveis" que entram e saem, interagindo entre si e com os "fixos", pois cada "equipamento bluetooth" traz embutido um chip que nada mais é que um microrrádio transceptor ("Bluetooth Radio") (KOBAYASHI, 2004).

Os dispositivos móveis estão sendo fabricados com diversas finalidades: interfaces com GPS, com tocadores de áudio e vídeo, com câmeras fotográficas digitais, com jogos eletrônicos e com placas de comunicação multiprotocolos sem fio, entre outros (MATEUS; LOUREIRO, 2004).

As aplicações da tecnologia móvel estão avançando a cada dia. Por exemplo, em estudos realizados por nutricionistas na redução alimentar de seus pacientes através da coleta de informações sobre alimentos consumidos (data, hora e quantidade) no momento da refeição, via celular (GARROZI; SILVA, 2007).

Também está sendo utilizado no gerenciamento de informações cadastrais de municípios, o que permite a manipulação e disponibilização de informações alfanuméricas importantes para um bom planejamento, o acesso às informações do cadastro e a atualização dos dados, além de contar com a possibilidade de georreferenciamento dos imóveis dos municípios (ANDREIS, 2006).

Outro exemplo de desenvolvimento tecnológico é o trabalho que vem sendo realizado entre o departamento de engenharia elétrica da UFPA (Universidade Federal do Pará) e a empresa Ericson, que estão desenvolvendo uma nova tecnologia capaz de garantir uma comunicação celular limpa em florestas densas, dentro dos elevadores, túneis para automóveis e até mesmo em avenidas fechadas por árvores, segundo Luz (2007).

A disponibilização de serviços e aplicações depende da criação de novas ferramentas e plataformas de desenvolvimento. Nesse processo, desafios existentes em sistemas tradicionais irão aparecer com uma conotação mais forte ainda, como usabilidade e segurança (MATEUS; LOUREIRO, 1998).

\section{MATERIAIS E MÉTODOS}

O trabalho foi desenvolvido no Laboratório de Geomática da Universidade Federal de Santa Maria (UFSM). Com o emprego da linguagem NSBasic, foi desenvolvido um sistema para Pocket PC que proporciona a coleta de dados de inventário florestal para florestas plantadas. O sistema está estruturado de forma a gerenciar os dados e informações oriundas do campo.

O banco de dados desse sistema foi moldado no MSAcess, com tabelas que permitem o armazenamento de dados provenientes do inventário florestal e dão suporte visual à interface do programa. $\mathrm{O}$ banco de dados é composto pelas tabelas projeto, parcelas, espécies_P e dados_ $P$.

O sistema foi moldado conforme orientação do manual de campo do inventário florestal realizado no estado do Rio Grande do Sul no ano de 2001. Foram abordados os elementos de caracterização do projeto, as características da parcela, os procedimentos de levantamento de vegetação, a classe natural de idade das árvores, as classes de valor, o grau de estoqueamento, o grau de cobertura e as características fitossanitárias da árvore. 
No software foram desenvolvidas ferramentas para registrar os projetos, cadastrar as espécies florestais, cadastrar as parcelas, registrar e georreferenciar as parcelas amostrais, registrar os elementos dendrométricos e de fitossanidade e ainda calcular o volume $\left(\mathrm{m}^{3} / \mathrm{ha}\right)$ e a área basal $\left(\mathrm{m}^{2} / \mathrm{ha}\right)$. Desenvolveram-se também funções que permitem obter valores da altura média $(\mathrm{m})$ e do diâmetro médio (cm).

\section{RESULTADOS}

Os aparelhos móveis disponíveis hoje no mercado auxiliam o desenvolvimento de trabalhos que exigem deslocamento, além de representarem uma ferramenta para substituição dos processos feitos em papel por aplicativos baseados em formulários, segundo Galvin (2004).

Dando seqüência a essas tendências de mercado, são apresentadas as funções do software desenvolvido neste trabalho. A figura 1 apresenta a tela de inicialização do software com as seguintes opções de links: cadastro de projeto florestal, cadastro de parcelas amostrais, registro de medições dendrometrias, processamento primário dos dados, cadastro de espécies florestais e interface com sistema GPS bluetooth.

Em cada unidade amostral é possível realizar a caracterização da área (projeto), sendo registrado o nome do projeto, a espécie e o tamanho da área em hectare (Figura 2).

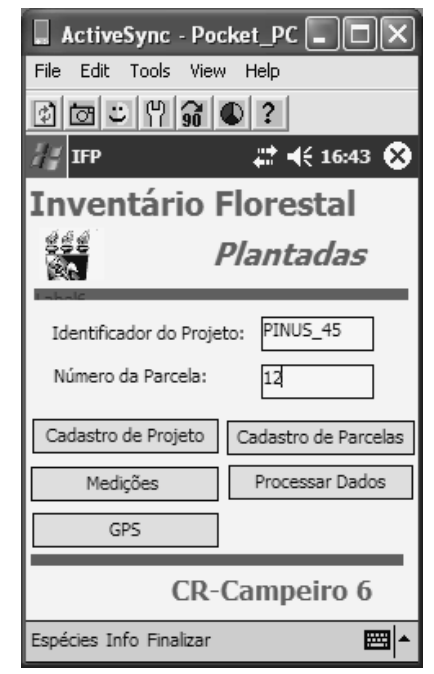

Figura 1. Abertura do sistema de coleta de dados para inventário florestal para florestas plantadas.

Figure 1. Data collecting system opening for planted forest inventory.

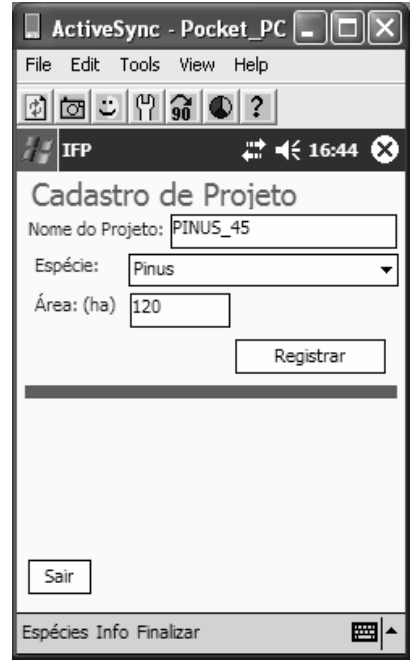

Figura 2. Cadastro do projeto para inventário florestal.

Figure 2. Project Record for Forest inventory.

Na figura 3, é mostrada a tela onde se realiza o cadastro de parcelas amostrais e as informações específicas para a área em estudo:

A) Identificação: nome do responsável pelo levantamento, o número da equipe, a hora de início da medição, espécie, área da unidade e coordenadas UTM do centro da unidade amostral.

B) Unidade amostral: número seqüencialmente da unidade amostral. No caso de parcelas permanentes, deve-se considerar o número definido na primeira ocasião.

C) Data de medição: indicar o mês e o ano da medição.

D) Espaçamento inicial: registrar a distância das árvores entre linhas e na linha.

E) Idade: registrar a idade real do povoamento em meses, levando em consideração o mês e o ano do plantio.

F) Tipo de parcela: permanente ou temporária. 


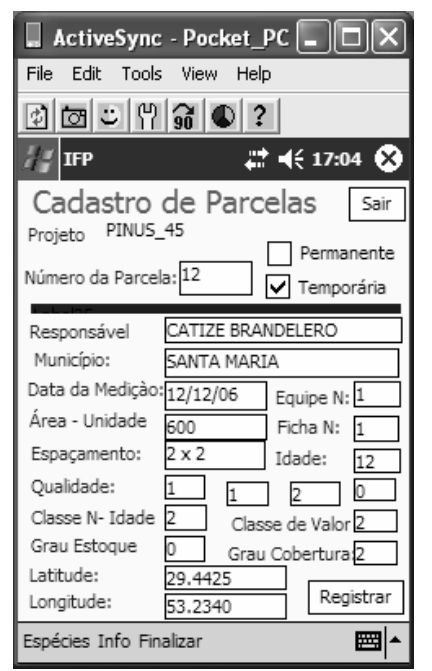

Figura 3. Tela para cadastro das parcelas amostrais no sistema de coleta de dados para inventário florestal de floretas plantadas.

Figure 3. Sampling plots Record in the data collecting system for planted forests Inventory.

Nessa mesma interface podem ser registradas as seguintes informações:

A. Qualidade das árvores:

- $\quad$ Forma do fuste (compreendida entre a base e o primeiro galho vivo):

0 . Não avaliado; 1 . Longo e reto; 2. Longo e irregular; 3. Médio e reto; 4. Médio e irregular; 4. Curto e reto; 5 . Curto e irregular.

- Galhos: 0. Não avaliado; 1. Grossos: diâmetro na inserção maior ou igual a 5,0 cm; 2. Finos: diâmetro menor que $5,0 \mathrm{~cm} ; 3$. Desramados: fuste que sofreu poda.

- Copas: 0. Não avaliada; 1 . Copa profunda: possui comprimento maior que $1 / 2 \mathrm{da}$ altura total da árvore; 2. Copa média: comprimento entre $1 / 2 \mathrm{e}^{1 / 4}$ da altura total da árvore; 3. Copa curta: comprimento inferior a $1 / 4$ da altura total da árvore.

- Defeitos: 0. Não avaliado; 1.Danos por animais: causados por roedores, cavalares, bovinos, etc; 2. Insetos; 3. Fungos; 4. Poluição; 5. Sem defeito.

B. Classe natural de idade (é registrado um dos códigos conforme a situação do estrato de desenvolvimento natural do povoamento):

$0 . \quad$ Não avaliado.

1. Estado jovem: compreende o período entre a implantação até o início do fechamento das copas.

2. Estado denso: povoamentos que se encontram no período entre o início do fechamento do coberto até o início dos desbastes.

3. Estado de desbaste: período que compreende o início dos desbastes até se atingir o diâmetro esperado.

4. Estado de madeira: povoamentos cujo CAP médio é maior que o diâmetro esperado.

C. Classe de valor (de acordo com o valor médio estimado da madeira do povoamento na referida unidade amostral), considerando:

$0 . \quad$ Não avaliada.

1. Altíssimo valor de produção: povoamentos que apresentam fustes longos e retos, sofreram desrama, apresentam copas curtas e árvores livres de defeitos.

2. Alto valor de produção: povoamentos que apresentam fustes longos e retos, galhos finos, copas curtas e árvores livres de defeitos.

3. Médio valor de produção: povoamentos que apresentam fustes longos e irregulares, e/ou médios e retos, e/ou curtos e retos, galhos finos e/ou grossos, copas médias e baixa incidência de defeitos (até $20 \%$ ). 
4. Baixo valor de produção: povoamentos que apresentam fustes médios e irregulares, e/ou curtos e retos, galhos grossos, copas grandes e defeitos em proporção superior a $20 \%$ e inferior a $60 \%$.

5. Nenhum valor de produção: povoamentos que apresentam fustes curtos e irregulares, galhos grossos e/ou finos, copas de qualquer tamanho e com alta incidência de defeitos $(>60 \%)$.

D. Grau de estoqueamento: relação existente entre o valor real e o volume obtido na tabela de produção, expresso em porcentagem (\%). Na primeira ocasião, não se avalia esse item.

E. Grau de cobertura: refere-se ao grau de cobertura do solo pelas copas das árvores:

1. Não avaliado.

2. Denso: copas que se entrelaçam.

3. Fechado: copas que se tocam na ponta dos galhos.

4. Aberto: copas distanciadas entre si de modo que uma segunda copa possa ocupar todo este espaço.

5. Claro: copas distanciadas entre si de modo que uma segunda copa não possa ocultar todo esse espaço.

6. Espaçada: copas distanciadas de tal forma que serão necessárias várias copas para ocultar esse espaço.

Já na tomada de dados das características fitossanitárias dos indivíduos, são observadas e registradas as informações individuais das árvores de uma determinada parcela previamente cadastrada (Figura 4):

A. Medida do DAP: registrar o DAP com precisão em milímetro.

B. Altura da árvore em metros.

C. Códigos (referem-se às características específicas de cada árvore):

- Primeiro campo: descreve as características das árvores: 0. sem peculiaridades; 1 . altura dominante (ho); 2. árvore morta; 3. árvores com desenvolvimento abaixo do limite de medição; 4. tronco bifurcado abaixo de $1,30 \mathrm{~m} ; 5$. tronco bifurcado acima de $1,30 \mathrm{~m} ; 6$. toco; 7 . árvore quebrada; 8. fuste danificado.

- $\quad$ Segundo campo: descreve as medidas de manejo aplicadas ou previstas para a árvore: 1 . sem peculiaridades; 2. marcada para desbaste; 3. árvore desramada; 4. árvore marcada para portasemente.

- $\quad$ Terceiro campo: descreve outras características que julgar necessárias: 0 . sem peculiaridades; 1 . falha; 2. árvore morta; 3 . árvore com desenvolvimento abaixo do limite de medição (CAP $\leq 16,0$ $\mathrm{cm}) ; 4$. toco; 5 . árvore com gonose; 6 . árvore atacada por vespa.

Além desses registros, são amostrados também elementos fitossanitários respectivos a cada indivíduo da parcela. Nessa rotina, o usuário registra o horário de início e fim das atividades na parcela.

$\mathrm{Na}$ figura 5, é apresentada a tela na qual o usuário pode processar a relação hipsométrica existente para a parcela a partir do modelo " $\mathrm{H}=\mathrm{b}_{0}+\mathrm{b}_{1}$.dap".

Com esse ajuste e a informação do fator de forma, é possível estimar o volume médio da parcela por hectare $\left(\mathrm{m}^{3}\right)$. Além dessas informações, são calculadas ainda a área basal $\left(\mathrm{m}^{2} / \mathrm{ha}\right)$, a altura média do povoamento e o dap (diâmetro médio à altura do peito) médio das árvores.

Isso possibilita que o técnico executor do inventário florestal, após concluir os trabalhos de campo, tenha possibilidade de uma avaliação rápida e confiável da situação da floresta em termos da parcela amostrada.

Devido ao sistema estar integrado com GPS Bluetooth, é possível também realizar a localização de parcelas florestais podendo inclusive ser utilizado no interior de povoamentos, pois a comunicação da posição do GPS pode atingir um raio de até $30 \mathrm{~m}$ de distância, segundo o fabricante. É apresentada na figura 6 a tela em que é visualizada a localização geográfica (sistema UTM) das parcelas amostradas ou de árvores dentro dos talhões.

Os dados levantados no sistema de coleta de dados em inventários florestais para florestas plantadas podem ser transferidos para uma planilha do software Excel ou para o módulo de inventário florestal do sistema CR Campeiro 6.0, para posterior processamento dos dados do inventário. 


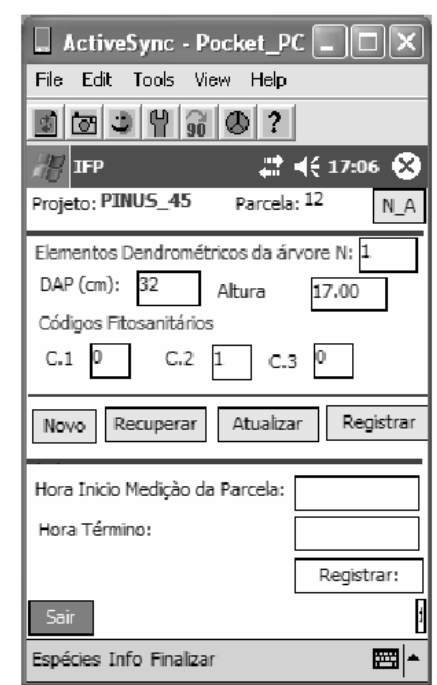

Figura 4. Tela de registro das medidas dendrométricas dos indivíduos amostrados.

Figure 4. Register of dendrometric measurements of sampled individuals.

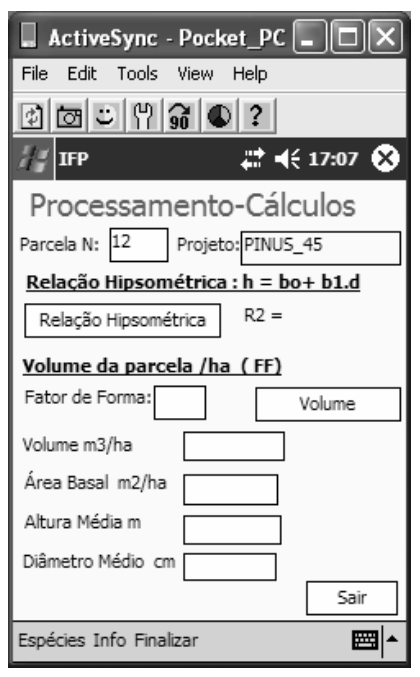

Figura 5. Tela de processamento primário dos dados para uma parcela.

Figure 5. Primary processing of data for one plot.

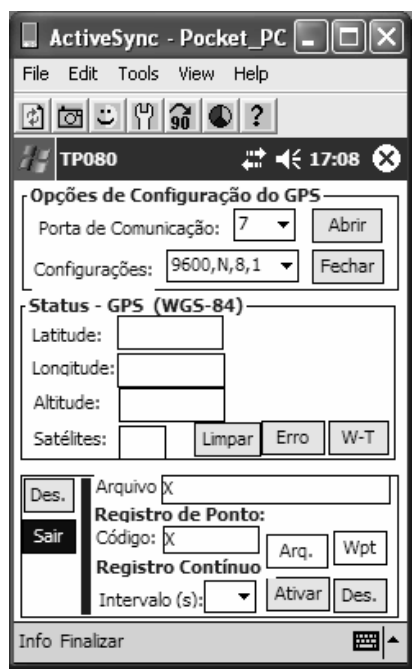

Figura 6. Tela de Interface com o aparelho GPS Garmim10 para localização geográfica.

Figure 6. Interface with the GPS Garmin 10 for geographical location.

\section{CONCLUSÕES}

O sistema de coleta de dados para inventários florestais de florestas plantadas, comparado com outros softwares desenvolvidos para Pocket $P C$, apresentou eficiência no armazenamento e processamento dos dados. Em conjunto com o GPS Bluetooth, mostrou-se eficiente na localização das unidades amostrais.

A mobilidade propiciada pelo Pocket PC permitiu que os dados do inventário fossem registrados e armazenados para posterior manipulação, propiciando, assim, rapidez e otimização das atividades no processamento dos dados após a transmissão para o computador, devido a não mais ser necessário digitar as informações oriundas de planilhas analógicas. 
A utilização de tecnologia móvel juntamente com o sistema de coletas de dados para inventário florestal de florestas plantadas é recomendado para melhorar a qualidade da quantificação e qualificação das florestas.

\section{REFERÊNCIAS}

ANDREIS, S. Descrição do sistema de recadastramento urbano municipal em implantação na cidade de Lajeado-RS. 62 p. Monografia (Especialização) - Universidade Federal de Santa Maria, Santa Maria, RS, 2006.

AVERY, T. E.; BURKHART, H. E. Forest Measurements. 4. ed. New York: McGraw Hill, 1994. 408 p.

BILLO, A. E. Uma pilha de protocolos Bluetooth adaptável à aplicação. $82 \mathrm{f}$. Monografia (Bacharelado em Ciência da Computação) - Universidade Federal de Santa Catarina. Florianópois, 2003.

GALVIN, D. Protótipo de sistema de CRM para dispositivos móveis utilizando a tecnologia .NET. 89 p. Trabalho de conclusão de curso (Bacharelado em Ciências da Computação) - Centro de Ciências Exatas e Naturais, Universidade Regional de Blumenau. Blumenau, 2004.

GARCIA, L. F. F.; LIMA, J. V. Sumarização baseada em contexto ambiental como fator de adaptação de objetos multimídia em dispositivos de computação móvel. Porto Alegre, 20003. Material didático da Universidade Federal do Rio Grande do Sul.

GARROZI, C.; SILVA, R. M. A. Uso da tecnologia móvel no auxílio à reeducação alimentar. Universidade Federal de Lavras. Lavras. Disponível em: $<$ http://pt.wikipedia.org/wiki/Tecnologia_m\%C3\%B3vel>. Acesso em: 27/01/2007.

HEWLLET PACKARD (HP). A mobilidade feita simplicidade. 2005. Catálogo. Disponível em: < http:// >. Acesso em: 04/01/2006.

HUSCH, B.; MILLER, C. I.; BEERS, T. W. Forest mensuration. New York: The Ronald, 1972. 410 p.

KOBAYASHI, C. Y. A Tecnologia Bluetooth e aplicações. Monografia (Computação Móvel) Universidade de São Paulo, São Paulo, 2004.

LOUREIRO, A. A. F.; SADOK, D. F. H.; MATEUS, G. R.; NOGUEIRA, J. M. S.; KELNER, J. Comunicação sem fio e computação móvel: tecnologias, desafios e oportunidades. In: CONGRESSO DA SOCIEDADE BRASILEIRA DE COMPUTAÇÃO, 23., 2003. Campinas. Disponível em: $<$ http://www2.dcc.ufmg.br/ loureiro/cm/docs/jai03.pdf> . Acesso em: 09/03/2006.

LUZ, E. Telefonia móvel sem interferência na Amazônia. Disponível em: $<$ http://www.ufpa.br/beiradorio/arquivo/beira37/noticias/noticia1.htm>. Acesso em: 16/02/2007.

MATEUS, G. R.; LOUREIRO, A. A. F. Introdução à computação móvel. Material didático do Departamento de ciências da computação da UFMG. 1998. p.115. Disponível em: $<$ http://www.di.inf.puc-rio.br>. Acesso em: 01/11/2005.

MATEUS, G. R.; LOUREIRO, A. A. F. Introdução à computação móvel. Material didático do Departamento de ciências da computação da UFMG. 2004. 120f. Disponível em: $<$ http://www2.dcc.ufmg.br/ loureiro/cm/docs/cm_livro_2e.pdf>. Acesso em: 09/03/2006.

PÉLLICO NETTO, S.; BRENA, D. A. Inventário Florestal. Curitiba: Ed. dos autores. 1997. 316 p.

PÉLICO NETTO, S. Métodos de amostragem em povoamentos florestais. Piracicaba: IPEF, 1984.

SANQUETTA, C. R.; WATZLAWICK , L. F.; CORTE, A. P. D.; FERNANDES, L. A. V. Inventários Florestais: planejamento e execução. Curitiba: Multi-Graphic, 2006. 270 p.

SEBEM, E. Desenvolvimento e aplicabilidade de uma base de dados relacional para o inventário florestal contínuo do Rio Grande do Sul. 69 f. Dissertação (Mestrado em Engenharia Agrícola) Universidade Federal de Santa Maria, Santa Maria, RS, 2000.

WATZLAVICK, L. F.; BRENA, D. A.; BOLFE, E. L.; MADRUGA, P. R. de A. Utilização de GPS na localização das unidades amostrais no Inventário Florestal Contínuo do Rio Grande do Sul. In: CONGRESSO FLORESTAL ESTADUAL DO RIO GRANDE DO SUL, 8., 2000. Nova Prata, Anais...Nova Prata: [s.n.], 2000. 1 CD-Room. 